\title{
Evidence based pediatric care closer to home: A mixed methods evaluation of a clinical pathways-based emergency outreach program
}

\author{
Mona Jabbour*1,2, Sarah Reid ${ }^{1,3}$, Danica Irwin ${ }^{3}$, Kristina Rohde ${ }^{3}$, Andrea Losier ${ }^{1}$, Eleanor Holmgren ${ }^{3}$, Nicholas \\ Barrowman $^{4}$, Katherine Moreau ${ }^{4}$ \\ ${ }^{1}$ Department of Pediatrics, Children's Hospital of Eastern Ontario, Ottawa, Canada \\ ${ }^{2}$ Pediatrics and Emergency Medicine, University of Ottawa, Ottawa, Canada \\ ${ }^{3}$ Children's Hospital of Eastern Ontario Research Institute, Ottawa, Canada \\ ${ }^{4}$ Clinical Research Unit CHEO Research Institute Ottawa, Ottawa, Canada
}

Received: October 6, 2016

DOI: $10.5430 /$ jha.v6n1p43
Accepted: December 12, 2016

Online Published: January 4, 2017

\begin{abstract}
Objective: While specialized pediatric care is not feasible in all Emergency Department (ED) settings, enhanced system capacity is needed to appropriately address pediatric emergency care needs. Our objective was to evaluate an innovative clinical pathway (CP) based regional ED outreach program within community EDs.

Methods: This mixed-methods design includes pre/post chart audits, qualitative interviews, ED site audits, and website utilization metrics. The study was conducted in community-based hospital EDs within the region. ED nurses, physicians and administrators from eight hospitals participated in the study. The ED outreach program involved implementation of 3 CPs addressing common pediatric emergency presentations: asthma, croup and gastroenteritis. Primary outcomes from chart audits included presence of a CP-related document filed in relevant patient charts and the proportion of patients who received appropriate care as per the severity-based CP recommendations. Secondary outcomes included documented assessments of condition severity, use of specific treatments and hospital metrics.

Results: ED health professionals value the outreach initiative and CPs. Site audits confirmed ED presence and knowledge of CP tools. However several adoption barriers were identified, including time, perceived threats to physician autonomy and infrequent opportunities for use. Chart audits demonstrated site-dependent, variable uptake of CP resources into ED workflows. In total, 1,274 health records were audited, with the following pre/post breakdowns: 203/211 (asthma), 131/193 (croup) and 291/245 (gastroenteritis). We could not evaluate our primary outcomes due to infrequent documentation of condition severity. Significant post-implementation improvements were demonstrated in gastroenteritis management. Median length of stay remained unchanged (asthma) or increased (croup, gastroenteritis). ED site was an important predictor of some asthma treatments and LOS for asthma $(p<.001)$, croup $(p=.01)$ and gastroenteritis $(p<.001)$.

Conclusions: CP documents were available in EDs, but may not be utilized as fully intended. Site heterogeneity may account for lack of significant change in several outcome variables.
\end{abstract}

Key Words: Clinical pathways, Emergency outreach, Pediatric emergency care, Program evaluation, Continuing medical education, Mixed methods evaluation

*Correspondence: Mona Jabbour; Email: Jabbour@ cheo.on.ca; Address: Department of Pediatrics, Children’s Hospital of Eastern Ontario; Pediatrics and Emergency Medicine, University of Ottawa, Ottawa, Canada. 


\section{INTRODUCTION}

Pediatric patients represent approximately $25 \%$ of Emergency Department (ED) visits in the United States and Canada ${ }^{[1,2]}$ with most visits occurring outside of specialized pediatric EDs. Approximately $75 \%$ of pediatric emergency care visits occur in general EDs, ${ }^{[1-4]}$ likely reflecting families seeking care closer to home. Regardless of where they present, all children deserve quality emergency care. This may be compromised due to the competing demands of general emergency medicine that compound evidence-topractice gaps across all medical fields. ${ }^{[5-11]}$ Because specialized pediatric systems of care are not feasible in all ED settings, calls have been made to increase capacity of existing systems and providers to appropriately address pediatric emergency care needs. ${ }^{[12,13]}$

We hypothesized that a targeted strategy to integrate evidence-based pediatric management recommendations into ED care systems would improve the evidence-to-practice gap within general community EDs. Towards this end, we implemented an innovative ED outreach program based on clinical pathways (CPs), which are tools designed to operationalize best evidence guidelines into accessible bedside formats for health provider teams. ${ }^{[14-20]}$ Developed by experts at the Children's Hospital of Eastern Ontario (CHEO), the CPs have been fully integrated into the care provided at this pediatric academic center. Our initial pilot included eight hospitals within our referral region, where pediatric patients comprise $15 \%-40 \%$ of visits. Beyond CHEO, regional pediatric expertise is sparse, with limited access to in-house pediatric consultants. We provided interprofessional education workshops and ancillary resources through a dedicated outreach website (https://outreach.cheo.on.ca) and engaged local champions to support CP use. Details of our ED outreach program and preliminary evaluation findings, including participant satisfaction and knowledge acquisition, are reported elsewhere. ${ }^{[21]}$

The purpose of this study was to conduct a more extensive evaluation of this innovative CP-based regional ED outreach program. This paper will report on: (1) reactions to, and (2) utilization of three pediatric CPs addressing three of the most common pediatric presentations - asthma, croup and gastroenteritis - within participating EDs.

\section{METHODS}

\subsection{Study design}

Our mixed methods evaluation design included use of pre/post chart audits; qualitative interviews and focus groups; ED site audits; and website utilization metrics (see Table 1). Research ethics board approval was secured at the CHEO Research Institute and each participating institution before initiation of any activities. Informed consent was obtained from all interview and focus group participants.

Table 1. Summary of the children's hospital ED outreach program evaluation analysis

\begin{tabular}{llll}
\hline & Participants' Reactions to the CPs & CP Use by ED Health Professionals & Study Sample Period \\
\hline \multirow{2}{*}{ Description } & Participant reactions to CP tools & $\begin{array}{l}\text { Adoption of CPs and/ or } \\
\text { CP-recommended management }\end{array}$ & $\begin{array}{l}\text { Implementation completion dates across } \\
\text { sites: April 2010 - Sept 2011 }\end{array}$ \\
& $\begin{array}{l}\text { Participant satisfaction surveys } \\
\text { Key Informant interviews }\end{array}$ & Key Informant interviews & April - July 2011 \\
& Focus Groups & Focus Groups & April - July 2011 \\
Data Source & & Pre/post chart audits & April 2009-Sept 2012 \\
& & ED site audits & May 2011 - Sept 2012 \\
& & Website utilization metrics & April 2008 - Sept 2013 \\
\hline
\end{tabular}

Note. ${ }^{*}$ As reported in previous manuscript ${ }^{[21]}$; CP: clinical pathway; ED: emergency department

\subsection{Study setting and population}

Study participants included emergency nurses and physicians working in one of eight community hospitals within our referral region.

\subsection{Study protocol and key outcome measures}

\subsubsection{Participants' reactions to CPS}

To assess issues within the organizational context, an administrative lead, defined as a nurse manager or physician chief, at each hospital was invited to participate in a semi-structured key informant interview. All eight sites agreed to an inter- view. At five sites two administrative leads were identified and interested to participate; therefore two interviews were conducted. In total, 13 interviews, 8 with nurse leads and 5 with physician leads. A single author (KR) with qualitative interviewing expertise conducted all interviews in English using a semi-structured interview guide. Interviews were audio-recorded and transcribed verbatim for analysis.

To assess how extensively the CPs had become part of the ED culture, we sought to conduct multi-disciplinary focus group sessions at each site, inviting nurses, physicians and other allied health professionals. In total, 8 focus group sessions, 
with up to 10 participants each, were held at four sites. Focus group participants were predominantly ED nurses. All focus group were conducted on site and in English by a single author (KR) with qualitative interviewing expertise. Focus groups were audio-recorded and transcribed verbatim for analysis. A focus group and/or key informant interview was conducted at each of the eight sites.

The site champion at each hospital assisted with local recruitment of participants for these interviews and focus groups. The latter were open to all staff and physicians at each hospital ED. No financial incentives were provided, although the focus group sessions involved a catered lunch. Participation was voluntary, with no penalties for refusal. Reasons for non-participation were not collected.

\subsubsection{CP use by ED health professionals}

We assessed CP use among ED health professionals using: (1) pre/post chart audits; (2) key informant interviews and focus group sessions, as described above; (3) ED site audits; and (4) website utilization metrics. An experienced research coordinator conducted the chart audits, using a data collection form created and piloted for each of the three clinical conditions. Using ICD-10 codes, all relevant charts were retrieved from the first week of each month during the twelve-month pre and post-implementation periods at each site. Chart abstractions were limited to those from patients that met defined inclusion/exclusion criteria for the relevant $\mathrm{CP}$; that is, only from patient charts for which use of the $\mathrm{CP}$ was appropriate. Information collected included patient demographics, assessment, management, discharge diagnosis, and disposition details. Our study outcomes are summarized in Table 2. Primary outcomes included presence of a CP-related document filed in the health record of relevant patients and the proportion of patients who received appropriate care, as per the severity-based CP recommendations. Secondary clinical outcomes included documentation of assessments and hospital metrics.

The research coordinator conducted post-implementation ED site audits concurrently with the chart audits. Using a written checklist, the research coordinator made observations and consulted with ED health professionals on duty to assess awareness, availability, and perceived usefulness of the CP tools. The ED Outreach website utilization assessment compared two matched 24-month periods, pre/postimplementation. Utilization metrics included website hits for specific pathway tools, including the pathway algorithms, pre-printed order sets, parent information resources, and drug calculation programs.

Table 2. Primary and secondary study outcomes

\begin{tabular}{|c|c|c|}
\hline \multirow{2}{*}{$\begin{array}{l}\text { Primary Process Outcome } \\
\text { Primary Clinical Outcome }\end{array}$} & \multicolumn{2}{|c|}{ Is the CP being used, based on a CP or Pre-Printed Order filed on the chart of relevant patients? } \\
\hline & $\begin{array}{l}\text { Proportion of pediatric patients who } \\
\text { received CP recommended care }\end{array}$ & $\begin{array}{l}\text { Asthma } \\
\text { - mild to moderate exacerbation treated with inhaled salbutamol } \\
\text { - moderate to severe exacerbation treated in ED with inhaled } \\
\text { salbutamol and systemic corticosteroids } \\
\text { - severe exacerbation treated with inhaled salbutamol and } \\
\text { ipratropium, and systemic corticosteroids } \\
\text { Croup } \\
\text { - dexamethasone for all patients } \\
\text { - severe croup treated with epinephrine } \\
\text { Gastroenteritis }\end{array}$ \\
\hline Secondary Clinical Outcomes & $\begin{array}{l}\text { 1. Documented severity assessment } \\
\text { for each condition }\end{array}$ & $\begin{array}{l}\text { Asthma: Pediatric Respiratory Assessment Measure (PRAM) score } \\
\text { Croup: Westley croup score } \\
\text { Gastroenteritis: Clinical estimation of dehydration (mild/mod/severe) }\end{array}$ \\
\hline & $\begin{array}{l}\text { 2. ED length of stay } \\
\text { 3. Hospital admission/ transfer } \\
\text { 4. Return ED visits (within } 1 \text { week) }\end{array}$ & \\
\hline
\end{tabular}

Note. CP: clinical pathway; ED: emergency department

\subsection{Data analysis}

\subsubsection{Quantitative}

For each condition, ED length of stay was found to be skewed; analyses were therefore conducted using logtransformed ED length of stay. This was compared between phases using mixed effects models, treating site as a random effect. To facilitate interpretation, results based on log-transformed quantities that are treated as normally distributed in the models have been transformed back to the original scale. Consequently, means on the log-scale must be 
interpreted as medians when transformed back to the original scale.

\subsubsection{Qualitative}

We used a conventional qualitative content analysis ${ }^{[22]}$ because minimal information and research literature exists on this topic area. This approach ensured an inductively derived coding scheme that flowed directly from the data. Two trained qualitative researchers (KM; KR) versed in this area first independently read all transcripts multiple times to obtain a sense of the whole. ${ }^{[23]}$ Next, they independently read through the data verbatim to derive an initial coding scheme, then sorted these codes into overarching categories based on how they related to one another. They met to compare their categorical systems and through discussion developed definitions for each category. Finally, one of the qualitative researchers reanalyzed the transcripts using this agreed list of categories and codes, identifying exemplars of each. The use of multiple coders in the development of categories and codes, combined with exemplars, ensured the rigor and trustworthiness of the analysis. An audit trail was kept of coding decisions, data analysis procedures and copies of evolving coding schemes. ${ }^{[24]}$

\section{Results}

\subsection{Participants' reactions to CPs}

We identified three major themes regarding participants' reactions to the CP tools:

\subsubsection{Theme 1: Standardization of care}

Participants from all sites described high satisfaction with CPs' perceived and potential impact in standardizing care; that $\mathrm{CP}$ adoption would promote uniformity and continuity of pediatric care across centres. For example, one participant described how,

"The pathways, I think, in our ER here has helped, you know, standardize everything. So, when we do send somebody to CHEO we are on the same path, right, of what we are using."

Another individual reiterated this, noting:

"If they used the pathways you would have a more consistent approach to care. Patients definitely receive the same standard so you would not have some people getting different levels of care."

Participants also discussed how standardization improves safety and minimizes medical error, especially among providers who treat children infrequently in the ED. As one participant stated:

"I do not get to spend a lot of consistent time there [in the ED] and the pathways provide me with an excellent opportunity to not miss things or make mistakes. They trigger the questions and the treatments that I need to be following through on, which is essential."

\subsubsection{Theme 2: Credibility}

Participants discussed the importance of establishing credibility of their ED in providing care. Alignment with CHEO's strong reputation is critical to this credibility. Interviewees relate implementation of the CHEO's CPs with enhancing their ED capabilities. One participant commented:

"I think because it is being labeled as CHEO's pathway it is giving us great strength... If it becomes an issue with a parent, we can say we are doing things the same way as they do at $\mathrm{CHEO}$, which is a big piece as well. The administration and everyone is happy to be able to attach a bit of a value label to it by saying it is CHEO's protocols."

\subsubsection{Theme 3: Interdependent practice}

Participants appreciated that CPs can be initiated by both physicians and nurses. Several expressed that they "like the idea of a triage nurse initiating the pathway".

They also described how CPs promote a collaborative environment where physicians and nurses work interdependently for the child's benefit. Reflecting her own experience, one participant said:

"I think using [the pathway] will improve all of that, definitely the flow. The fact that you start treating a dehydrated patient right away; a lot of these kids will feel better before they even see the physicians."

\subsection{Use of CPs by ED health professionals}

\subsubsection{Pre/post chart audits}

In total, 1,274 patient health records were audited pre/postintervention for three clinical conditions: asthma, croup and gastroenteritis (see Table 3). Based on lack of a physical $\mathrm{CP}$ or associated pre-printed order set, $\mathrm{CP}$ documents were infrequently found within the patient records. Regardless of whether a CP document was used, the CP education sessions instructed participants to assess condition severity by using a validated scoring tool (asthma, croup) or clinical signs (gastroenteritis). Despite this, severity assessments of any type were infrequently documented in the charts. As such, because CP treatment recommendations are based on condition severity, we could not assess appropriateness of patient-specific management.

Controlling for site, significant differences were found in pre/post use of specific treatments for gastroenteritis with increased use of oral rehydration therapy and ondansetron, and decreased use of dimenhydrinate (see Table 4). 
Table 3. Descriptive statistics for pre/post chart audits

\begin{tabular}{|c|c|c|c|c|c|c|}
\hline & \multicolumn{2}{|c|}{ Asthma $(n=414)$} & \multicolumn{2}{|c|}{ Croup $(n=324)$} & \multicolumn{2}{|c|}{ Gastroenteritis $(n=536)$} \\
\hline & Pre & Post & Pre & Post & Pre & Post \\
\hline Pre/Post Implementation (n) & 203 & 211 & 131 & 193 & 291 & 245 \\
\hline Mean Age in Years $(S D)$ & $8.38(5.45)$ & $7.94(5.00)$ & $3.20(2.55)$ & $3.07(2.31)$ & $5.03(4.73)$ & $4.38(4.58)$ \\
\hline CP Document filed in Chart (n [\%]) & $0(0.0)$ & $13(6.2)$ & $0(0.0)$ & $33(17.1)$ & $0(0.0)$ & $4(1.5)$ \\
\hline
\end{tabular}

Note. $S D$ : standard deviation

Table 4. Use of specific treatments pre vs. post pathway implementation

\begin{tabular}{llllll}
\hline Gastroenteritis & $\mathbf{n}$ & OR & Lower & Upper & $\boldsymbol{p}$-value \\
\hline ORT & 536 & 3.77 & 1.90 & 7.48 & $<.001$ \\
Ondansetron & 536 & 162.86 & 20.20 & $1,313.05$ & $<.001$ \\
Dimenhydrinate & 536 & 0.24 & 0.13 & 0.44 & .001 \\
IV Fluids & 536 & 1.07 & 0.57 & 2.02 & .96 \\
Croup & & & & 2.29 & .57 \\
$\begin{array}{l}\text { Dexamethasone } \\
\text { Asthma }\end{array}$ & 324 & 1.25 & 0.68 & & .50 \\
Systemic Corticosteroid & 413 & 1.18 & 0.78 & 1.81 & \\
\hline
\end{tabular}

Note. ORT: oral reydration therapy; OR: odds ratio; Severity-based management could not be assessed due to incomplete documentation in patient health records

Table 5. ED length of stay by condition

\begin{tabular}{|c|c|c|c|c|c|c|}
\hline & \multicolumn{2}{|c|}{ Asthma } & \multicolumn{2}{|c|}{ Croup } & \multicolumn{2}{|c|}{ Gastroenteritis } \\
\hline & Pre & Post & Pre & Post & Pre & Post \\
\hline $\begin{array}{l}\text { Median length of } \\
\text { stay hours (IQR) }\end{array}$ & $\begin{array}{l}1.73 \\
(1.01-2.64)\end{array}$ & $\begin{array}{l}1.70 \\
(1.08-2.97)\end{array}$ & $\begin{array}{l}1.17 \\
(0.7-1.88)\end{array}$ & $\begin{array}{l}1.52 \\
(0.78-2.62)\end{array}$ & $\begin{array}{l}1.45 \\
(0.75-2.75)\end{array}$ & $\begin{array}{l}2.27 \\
(1.16-3.61)\end{array}$ \\
\hline $\begin{array}{l}\text { ED Site was an in } \\
\text { Admissions/Tran } \\
\text { ED Revisits: No }\end{array}$ & $\begin{array}{l}\text { Nant predictor } \\
\text { No significa } \\
\text { ficant differe }\end{array}$ & $\begin{array}{l}\text { length of stay } \\
\text { lifference Pre } \\
\text { Pre vs. Post I }\end{array}$ & $\begin{array}{l}\text { Ima }(p<.00 \\
\text { Interventio } \\
\text { ion }\end{array}$ & oup $(p=.0$ & Gastroenterit & $<.001)$ \\
\hline
\end{tabular}

Note. IQR = inter-quartile range; ED: emergency department

Between sites, several management practices varied significantly for asthma, including salbutamol administration by nebulization only $(p=.04)$ and mean number of salbutamol treatments $(p=.001)$. Regarding other secondary clinical outcomes, median ED length of stay post-implementation was unchanged (asthma) or increased (croup, gastroenteritis) (see Table 5). Note, the croup CP recommendation included a two-hour observation post-epinephrine treatment. Again, ED site was a significant predictor of ED length of stay for asthma $(p<.001)$, croup $(p=.01)$, and gastroenteritis $(p<.001)$. Hospital admissions, transfers or return visits were too infrequent to detect significant differences pre/post implementation.

\subsubsection{Qualitative interviews}

As reported by administrators and frontline ED nurses and physicians, uptake of $\mathrm{CP}$ resources into ED workflows was dependent and variable. We identified four major themes influencing use of the CPs.

Published by Sciedu Press
(1) Health professionals' perceptions and characteristics: Participants identified how their perceptions of medical practice and personal characteristics influenced whether they embraced the CPs. For instance, one participant stated:

"We have not been using the pathways because when they were presented at the Emergency Committee, the physicians agreed with some of them, but not all of them ..."

Participants also suggested that $\mathrm{CP}$ use might be contingent on health professionals' age and willingness to integrate technology into their practices, as evidenced below:

"This may be a negative for you per se, but I would say that in our facility because we have not had pathways and we have basically an older physician group with a reluctance to adopt new technology and new ways of thinking, that this older group has been resistant in adopting both the pathways and pre-printed orders... However, recent graduates in emergency, specifically, those who have entered in about the last ten year have incorporated [these] as the mainstream 


\section{practice.”}

(2) Physician autonomy: Participants discussed the importance of evidence-informed decision-making in optimizing patient outcomes. However, they expressed concern that a perceived lack of physician autonomy in deciding best management for their patients might be hindering CP use. As one interviewee stated:

"Physicians are ordering treatments that are very consistent with the pathways, but I do not believe they are implementing the actual pathways. I do not know if they feel that the autonomy of decision-making is removed from their hands now."

Another commented on how,

"there are some physicians who are still cowboys. They like to do things their own way. ... Some of them do not want to do the protocols and they tend to make their own decisions."

(3) Time: Participants noted that, while CPs are desirable, additional time is required to integrate them into the care process, as supported in the following quotations:

"For us to roll out something takes a long time. We probably have 12 or 15 people that float, too. They will just do a shift and leave, but there is nobody really in charge of, you know, physician education. There is nobody who is here five days a week or anything."

"I understand that it is a slow process and that we have many new people on our staff to bring up to speed on those. So, the pathways, I would say, the use rate is probably fifty percent at best."

Participants also highlighted that $\mathrm{CP}$ use requires more devoted time for reading and completing necessary paperwork, reflecting statements such as,

"It does seem to slow you down, it does take time, you know to go through it",

and "I do not have time to hunt down everybody and talk to them to have them fill out the [education] form and sign off on it. So, that has been the big limiting step."

However, others emphasized how additional CP education helped participants realize they are actually easy to use, reflecting one clinician's statement,

"Oh, that is so simple. But when you do not have the training at first you are thinking that this very difficult, but it is, really, simply checking it off."

and "... from a physician point of view, they are easy to use ... I could take that and use it tomorrow."
(4) Opportunity for CP use: Lastly, participants commented on their limited opportunities for $\mathrm{CP}$ use, which may have impacted integration into their routine practices. Explaining this situation, one interviewee stated:

"We don't have the opportunity to use them, we don't have the acuity to use them that often they forget that they're there".

Nonetheless, one participant expressed confidence that after "they have used it once or twice, then they are okay. ... and [that] when we use them, the more familiar we will become."

\subsubsection{ED site audits}

Post-implementation site audits were completed in seven EDs, with input from health professionals (two nurses, one physician) on duty in each ED during the visit. All sites had a visible outreach poster and pre-printed CHEO health education documents. The health professionals interviewed at each site knew the location and purpose of the $\mathrm{CP}$ documents. They reported the CP documents as moderately to highly useful. The ED outreach website was bookmarked on computers in $4 / 7$ (57.1\%) sites visited.

\subsubsection{Website utilization}

Over the course of the study period, the CHEO ED Outreach Website became broadly available beyond our 8 pilot sites. Consequently, a disproportionate increase in the number of non-study site users accessing the website in the postimplementation period precluded our ability to differentiate data among sites. Website utilization increased overall in the post-implementation period. However, without accurate data specific to our sites, a decision was made to disregard this analysis.

\section{Discussion}

This study demonstrated that ED health professionals value the CP-based ED outreach program that, as they see, "enhances [their] capacity to deliver good care". CPs promote interprofessional work and interdependence, empowering nurses to initiate care sooner. Our mixed-methods evaluation was essential in assessing specific outcomes through quantitative measures and, more importantly, understanding these outcomes through focus group and key informant interviews. Qualitative data revealed that although the CPs were highly valued, adoption barriers persisted. Specifically cited barriers included change readiness, perceived threats to physician autonomy, time to integrate into workflows, and infrequent opportunities for use. Based on our ED site audits, awareness and physical presence of the CPs were not an issue. Additionally, although we heard the CPs were being used, we could not assess management appropriateness post-implementation because condition severity was 
infrequently documented in patient records. We found significant changes in accordance with CP-recommended therapies for gastroenteritis, but minimal to no significant changes in therapies aligned with the asthma and croup CPs. These differences may be due to several factors, such as minimal physician participation in educational sessions, favorable benefit-risk ratios for some treatments (gastroenteritis $\mathrm{CP}$ ), and a pre-implementation ceiling effect in current management (croup). The increased croup ED length of stay may represent improved CP-recommended care delivery.

Of note, ED site was a significant predictor for use of specific treatments and ED length of stay in all three conditions. Although we were not powered to evaluate site-specific practice changes, these findings further highlight the concerning variability in care for common conditions. ${ }^{[7-11]}$

There is growing evidence for $\mathrm{CP}$ effectiveness, including improved safety, quality, cost savings and other efficiencies. $^{[14-20,25-27]}$ Most pediatric CP studies reporting positive outcomes were done in single pediatric institutions where implementation may have been less challenging. Negative CP trials typically result from incomplete implementation. ${ }^{[25]}$ Our program sought to guide implementation of expertdeveloped pediatric CPs in general ED settings. These are complex interventions requiring multimodal interventions, frequent reinforcements and sufficient time for established change in organizational practices. Targeted initiatives to improve physician engagement may have also led to more successful CP implementation.

A key strength of this study was our pragmatic intervention design that would guide sites with necessary tools and approaches for successful CP implementation. More direct participation by study personnel with local implementation activities may have resulted in greater success. However this would limit generalizability as this approach does not address real-life challenges and could misinform application of study recommendations. ${ }^{[28]}$ Another strength was our mixedmethods evaluation approach that sought to identify potential explanations for our findings through qualitative data. Understanding the issues behind incomplete implementation provides greater insights for eventual success.

\section{Limitations}

There are some limitations to our study. Our health region is unique, with sparse pediatric consultant availability beyond our academic referral centre. Local pediatricians, if present within given sites, may have facilitated local implementation processes by advocating for improved pediatric care. Another limitation relates to the participants attending education sessions and focus groups, who tend to be more committed and may not represent views of more change-resistant colleagues. Physicians participated infrequently in these interventions, which is unfortunate given their prominent roles in ED teams.

\section{Conclusions}

$\mathrm{CP}$ implementation is a complex intervention and requires sufficient time and resources to establish the change process. Although we were able to demonstrate that $\mathrm{CP}$ documents were available within EDs and valued by ED health professionals, they may not be used as fully intended. Site heterogeneity may account for the lack of significant change on several outcome variables. Future research should explore physician-targeted initiatives for more successful implementation.

\section{ACKNOWLEDgements}

The ED Outreach Initiative was funded by the Provincial Ministry of Health and Long Term Care. The program evaluation was funded by the Children's Hospital Academic Medical Organization Innovation Fund. This project was approved by the Children's Hospital Research Ethics Board (protocol number blinded), as well as by Research Ethics Boards or Ethics Committees at all of the sites.

\section{Conflicts of InTEREST Disclosure}

The authors report no conflict of interest.

\section{REFERENCES}

[1] Canadian Institute for Health Information. Emergency Departments and Children in Ontario. Accessed October 28, 2015. Available from: https://secure.cihi.ca/free_products/aib_apr24 -08_en.pdf

[2] Rasooly IR, Mullins PM, Alpern ER, et al. US Emergency Department Use by Children, 2001-2010. Ped Emerg Care. 2014; 30: 602607. https://doi.org/10.1097/PEC.0000000000000204

[3] Prentiss KA, Vinci R. Children in emergency departments: who should provide their care? Archof Dis Child. 2009; 94(8): 573-576.

\section{https://doi.org/10.1136/adc. 2008.137885}

[4] Gausche-Hill M, Schmitz C, Lewis RJ. Pediatric preparedness of United States emergency departments: a 2003 survey. Pediatrics. 2007; 120(6): 1229-1237. https://doi.org/10.1542/peds.2 006-3780

[5] Lang ES, Wyer PC, Eskin B. Executive Summary: Knowledge Translation in Emergency Medicine: Establishing a Research Agenda and Guide Map for Evidence Uptake. Acad Emerg Med. 2007; 14(11): 915-918. https ://doi .org/10.1111/j.1553-2712.20 07.tb02364.x 
[6] Lenfant C. Clinical research to clinical practice-lost in translation? N Engl J Med. 2003; 349: 868-74. https ://doi.org/10.1056/ NEJMsa035507

[7] Lang ES, Wyer PC, Haynes RB. Knowledge Translation: Closing the Evidence-to-Practice Gap. Ann Emerg Med. 2007; 49: 355-363. https://doi.org/10.1016/j . annemergmed.2006.08.022

[8] Goodman DC. Unwarranted variation in pediatric medical care. Pediatr Clin North Am. 2009; 56(4): 745-55. https ://doi.org/10.1 016/j.pcl.2009.05.007

[9] Dharmar M, Marcin JP, Romano PS, et al. Quality of care of children in the emergency department: association with hospital setting and physician training. J Pediatr. 2008; 153(6): 783-9. https://doi.org/10.1016/j.jpeds.2008.05.025

[10] Lougheed MD, Garvey N, Chapman KR, et al. Variation and gaps in management of acute asthma in Ontario emergency departments. Chest. 2009; 135: 724-736. https://doi.org/10.1378/chest . 08-0371

[11] Freedman SB, Gouin S, Bhatt M, et al. Prospective Assessment of Practice Pattern Variations in the Treatment of Pediatric Gastroenteritis. Pediatrics. 2011. https ://doi .org/10.1542/peds. 2010-2 214

[12] Kurtin P, Stucky E. Standardize to excellence: improving the quality and safety of care with clinical pathways. Pediatr Clin North Am. 2009; 56(4): 893-904. https ://doi.org/10.1016/j.pcl. 2009 .05 .005

[13] American Academy of Pediatrics - Committee on Pediatric Emergency Medicine, American College of Emergency Physicians - Pediatric Committee, Emergency Nurses Association - Pediatric Committee. Joint Policy Statement - Guidelines for Care of Children in the Emergency Department. Pediatrics. October 2009; 124(4): 1233 1243. https://doi .org/10.1542/peds. 2009-1807

[14] Rotter T, Kinsman L, James E, et al. Clinical pathways: effects on professional practice, patient outcomes, length of stay and hospital costs. Cochrane Database Syst Rev (Online). 2010; 3. Cochrane AN: CD006632. https://doi.org/10.1002/14651858.CD006 $632 \cdot$ pub2

[15] Vanhaecht K, De Witte K, Panella M, et al. Do pathways lead to better organized care processes? J Eval Clin Pract. 2009; 15: 782-788. https://doi.org/10.1111/j.1365-2753.2008.01068.x

[16] Browne GJ, Giles H, McCaskill ME, et al. The benefits of using clinical pathways for managing acute paediatric illness in an emergency department. J Qual Clin Practice. 2001; 21: 50-55. https://doi .org/10.1046/j.1440-1762.2001.00405.x

[17] Kozer E, Scolnik D, MacPherson A, et al. Using a preprinted order sheet to reduce prescription errors in a pediatric emergency department: a randomized, controlled trial. Pediatrics. 2005; 116: 1299 1302. https : //doi .org/10.1542/peds . 2004-2016

[18] McCue JD, Beck A, Smothers K. Quality toolbox: clinical pathways can improve core measure scores. J Healthc Qual. 2009; 31(1): 43-50. https ://doi .org/10.1111/j.1945-1474.2009.00008.x

[19] Vanhaecht K, Bollmann M, Bower K, et al. Prevalence and use of clinical pathways in 23 countries - an international survey by the European Pathway Association. Journal of Integrated Care Pathways. 2006; 10(1): 28-34. https ://doi.org/10.1258/j.jicp. 2006. 124

[20] Kinsman LD, Buykx P, Humphreys JS, et al. A cluster randomized trial to assess the impact of clinical pathways on AMI management in rural Australian emergency departments. BMC Health Serv Res. 2009; 9: 83. https://doi.org/10.1186/1472-6963-9-83

[21] Jabbour M, Reid S, Irwin D, et al. Clinical Pathway-Based Pediatric Emergency Outreach Program: Implementation and Preliminary Evaluation. CJHP. 2014; 67(3): 240-5.

[22] Hsieh H, Shannon S. Three Approaches to Qualitative Content Analysis. Qual Health Res. 2005; 15(9): 1277-1288. https : //doi.or g/10.1177/1049732305276687

[23] Tesch R. Qualitative research: Analysis types and software tools. Bristol, PA: Falmer; 1990.

[24] Rodgers B, Cowles K. The qualitative research audit trail: A complex collection of documentation. Nurs Health. 1993; 16(3): 219-226. https : //doi.org/10.1002/nur.4770160309

[25] Simmons J, Kotagal UR. Reliable implementation of clinical pathways: what will it take-that is the question. J Pediatr. 2008; 152(3): 303-4. https ://doi.org/10.1016/j.jpeds.2007.12.017

[26] Norton SP, Pusic MV, Taha F, et al. Effect of a clinical pathway on the hospitalization rates of children with asthma: a prospective study. Arch Dis Child. 2007; 92: 60-6. https ://doi.org/10.1136/ad c. 2006.097287

[27] Cunningham S, Logan C, Dunn MJG, et al. Effect of an integrated care pathway on acute asthma/wheeze in children attending hospital: cluster randomized trial. J Pediatr. 2007; 152: 315-20. https://doi.org/10.1016/j.jpeds.2007.09.033

[28] Wears RL. Lost in Translation. Ann Emerg Med. 2008; 51: 78-79. https://doi.org/10.1016/j.annemergmed.2007.06.015 